\title{
Management of intertrochanteric fractures by dynamic hip screw VS proximal femoral nail
}

\author{
N Brahma Chary ${ }^{1,}$, Bachu Srinivas ${ }^{2}$, Ajay Kumar Pandey ${ }^{3}$, Narayana Prasad ${ }^{4}$ \\ ${ }^{1,2}$ Associate Professor, ${ }^{3}$ Assistant Professor, ${ }^{4}$ Professor, Dept. of Orthopaedics, ${ }^{1,3,4}$ Shadan Institute of Medical Sciences, \\ Himayatsagar Rd, Hyderabad, ${ }^{2}$ Government Medical College, Nizamabad, Telangana, India
}

*Corresponding Author:

Email: nandakadichary@gmail.com

\begin{abstract}
Introduction: Inter trochanteric fractures are more common in the age group of 20 years to 60 years due to high velocity road traffic accidents, Whereas inter trochanteric fractures may also be seen in elderly people due to mild to moderate severity of trauma in addition to high velocity trauma. The management of stable intertrochanteric fractures in young and old individuals either by Dynamic Hip Screw (DHS) or by Proximal Femoral Nail (PFN) and management of unstable IT fractures by PFN in both the age groups young and old.

Materials and Methods: The study is conducted in the department of orthopaedics at Shadan Institute of Medical Sciences (SIMS). A total no. of 43 patients are included in the study out of which 20 are stable fractures, remaining are unstable fractures. The mean age is 34 years (range $20-60$ yrs). The mean follow up period is 34 months (12-36 months).

Results: Out of 20 patients with stable fractures treated by DHS, 15 pts (\%) are excellent outcome, 3pts (\%) good, 2 pts (\%) fair, poor result is nil. Out of 23 patients with unstable IT fractures treated by PFN, 18 pts (\%) are excellent, 3 pts (\%) good, 1 pt (\%) fair \& 1 pt (\%) with poor result.

Conclusion: All IT fractures which are stable in young and old patients can be treated either by DHS or PFN with satisfactory outcome and all unstable fractures in young and elderly people should be treated by PFN. Irrespective of type of fracture, fixed by PFN has got the advantage of early mobilisation and weight bearing.
\end{abstract}

Keywords: Proximal Femoral Nail, Dynamic Hip Screw, Intertrochanteric fractures, Osteoporosis, Derotation screw.

\section{Introduction}

The incidence of IT fractures is increasing every year significantly because of RTA and industrial accidents in young adults but in old people because of increased life expectancy and osteoporosis the IT fractures are increasing due to less severe / trivial trauma. ${ }^{4}$

For stable fractures with good quality of bone either DHS or PFN give good results, but in unstable, comminuted and osteoporotic bones, shortening of the limb, external rotation deformity, implant cut outs and reoperations are common occurance with DHS. ${ }^{7}$ Cephalomedullary device is currently prefered for these fractures but the present versions of intramedullary nails are also associated with technical and mechanical complications like cut out of the screws from the head. ${ }^{1}$ Prosthetic replacement using cemented implants is another alternative in the management of unstable osteoporotic fractures which allows early mobilization. ${ }^{5}$

\section{Materials and Methods}

This is a prospective study of 43 patients with 20 stable intertrochanteric fractures treated by DHS (12pts) and PFN (8pts) and 23 unstable fractures, all are treated by PFN only, during the period from Nov 2014 to June 2017 at SIMS. 3 patients were lost to follow up. The inclusion criteria are AO IT fracture types 2.2 and A 2.3 stable and unstable fractures, All young and old people of high velocity trauma, osteoporotic fractures due to mild to moderate violence, age group 20-60 years and 1-9 days old fractures. ${ }^{3}$ Exclusion criteria patients with pathological fractures, patients with contralateral fractures, older fractures more than 9 days, subtrochanteric fractures, basicervical fractures, grossly comminuted greater trochanteric fractures, patients with major comorbid conditions, patients who are unfit from anesthetic point of view and paediatric age group. ${ }^{6}$

Table 1: Incidence of sex

\begin{tabular}{|l|c|c|}
\hline Sex & $\begin{array}{c}\text { No. of } \\
\text { patients }\end{array}$ & Percentage \\
\hline Male & 30 & $66.66 \%$ \\
\hline Female & 15 & $33.33 \%$ \\
\hline Total & 45 & $100 \%$ \\
\hline
\end{tabular}

There are 30 male patients and 13 female patients. The age group ranged from $20-60$ years with mean age of 34.5 years, the medical comorbidities are diabetes, hypertension, hypothyroidism and ischemic heart disease in 13 patients.

Table 2: Incidence of age

\begin{tabular}{|l|c|c|}
\hline Age in years & No of patients & Percentage \\
\hline $20-29$ & 8 & $17.77 \%$ \\
\hline $30-39$ & 10 & $22.22 \%$ \\
\hline $40-49$ & 12 & $26.66 \%$ \\
\hline $50-59$ & 10 & $22.22 \%$ \\
\hline $60-69$ & 5 & $11.11 \%$ \\
\hline Total & 45 & $99.98 \%$ \\
\hline
\end{tabular}


All the patients were operated within 48 hours after presentation to the hospital, after investigations and getting fitness from physician, cardiologist and anesthesiologist and correcting anaemia in few patients, under $\mathrm{C}$-arm control on fracture table, with average surgery time of 75 minutes with minimal blood loss. Cefparazone / Sulbactum combination was used for antibiotic prophylaxis in a dose of $1 \mathrm{gm} 12$ hourly for a period of 3 days postoperatively, the first dose administered at the time of induction of anesthesia.

Table 3: Aetiology

\begin{tabular}{|l|c|c|}
\hline Cause & $\begin{array}{c}\text { No. of } \\
\text { patients }\end{array}$ & Percentage \\
\hline RTA & 38 & $84.44 \%$ \\
\hline Fall due to slip & 5 & $11.11 \%$ \\
\hline Fall from height & 1 & $2.22 \%$ \\
\hline Assault & 1 & $2.22 \%$ \\
\hline Total & 45 & $99.99 \%$ \\
\hline
\end{tabular}

All the stable IT fractures are fixed by closed reduction and internal fixation by DHS / PFN. In case of DHS guidewire is placed either postero inferior or central, entry point not below the level of lesser trochanter to avoid stress riser effect. In cases of PFN (short) taken care of entry points either tip of greater trochanter/just medial to it. ${ }^{4}$ All the unstable IT fractures are held by $\mathrm{K}$-wires after reduction. Before passing the guidewire reduction is checked under $\mathrm{C}$ arm in both AP and LAT views. ${ }^{9}$ In all cases tip apex distance (TAD) is maintained at less than $20 \mathrm{~mm}$. The average surgery time is 75 mins and average blood loss is $100 \mathrm{ml}$. Only 5 patients were given deep vein thrombosis (DVT) prophylaxis by low molecular weight Heparin for or 3 days, first dose 8 hours after surgery, along with calf compression device (DVT pump) for 48 hours post operatively. ${ }^{8}$ The patients were mobilised on 2 nd postoperative day allowing partial weight bearing with aid of a walker only in PFN cases, whereas the weight bearing is delayed for 4 weeks in cases of DHS. We took little more time for PFN cases. ${ }^{6}$

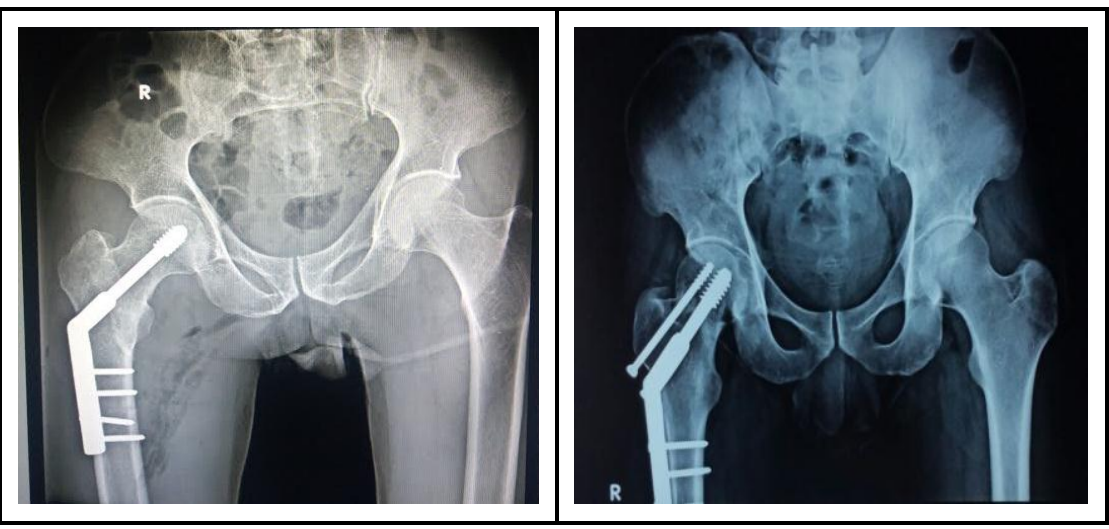

1. Stable IT Fracture fixed with DHS and Derotation screw

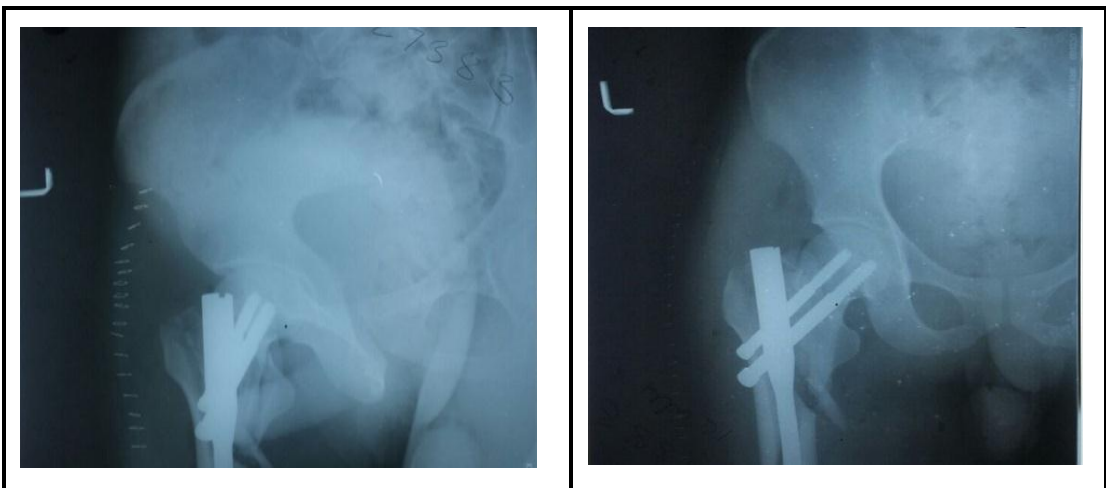

2. Comminuted unstable IT Fracture with subtrochanteric extension fixed with PFN in good alignment 


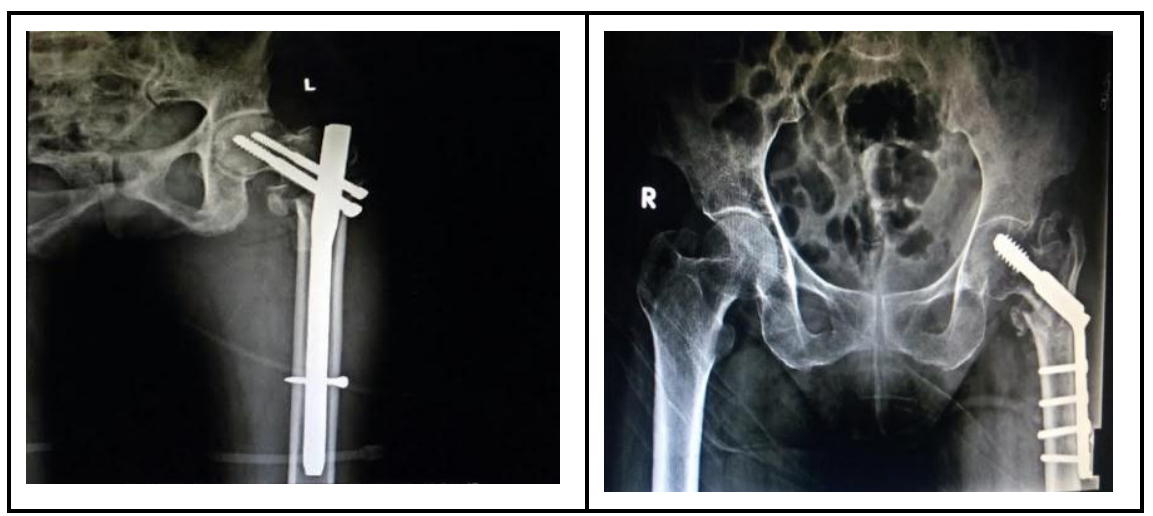

3. IT Fracture fixed with DHS and PFN are in varus position led to failure

Suture removal was done on 10th postoperative day, patients were reviewed at 1 month, 3 months, 6 months, 1 year and later examined on yearly follow up basis. At each follow up examined clinically and radiologically for hip / groin pain, thigh pain, shortening, implant loosening/bending, gait problem and Harrison Hip Score (HHS), implant position, migration / backing out of screws, varus collapse and rotation of head in cases of DHS with no anti rotation screw. HHS was calculated and patients are graded as poor $(<70)$, fair (70-79), Good (80 -89) and excellent $(90-100) .^{3}$

\section{Results}

The mean follow up time is 24.5 months (range 1236 months). The mean operative time was 75 minutes (range 75 - 90 mins). The mean intraoperative blood loss was $100 \mathrm{ml}$ (range $60-200 \mathrm{ml}$ ). There are no intraoperative complications. ${ }^{2}$ Complications in follow up period are proximal migration of screws into hip joint, backing out of screws, superior cut out of the screws, varus collapse, shortening of the limb, and superficial decubitus ulcer in one patient. ${ }^{4}$ The mean HHS at 6 months was 84.5 out of 43 patients 33 patients (\%) excellent, 8 patients (\%) good, 4 patients (\%) fair and 1 patient (\%) poor. 38 patients are walking independently and remaining are walking with cane. ${ }^{8}$ There are no incidences of implant bending or breakage, but occasional varus collapse with migration / cut through of screws in our study.

\section{Discussion}

IT fractures are traditionally treated with internal fixation using DHS / PFN. Union rates of close to $100 \%$ have been achieved in stable, well fixed fractures in patients with good quality of bone. However problems arise in unstable osteoporotic fractures where a high incidence of complication has been observed. Shortening of limb is a common complication in unstable fractures fixed with PFN with varus collapse. The complication rate is minimal or nil in unstable IT fractures fixed with PFN, which are anatomically reduced and held by $\mathrm{K}$-wires before internal fixation.

\section{References}

1. Campbell's Operative Orthopaedics. Vol II, 8th edition; Fracture of Hip \& Pelvis 898 - 907; Cephalomedullary device 911-913.

2. Outcome of intertrochanteric fractures treated by IM nail with 2 integrated lag screws-A study in asian population. Indian Journal of Orthopaedics: Vol 49 - issue 4, July August 2015;336-40.

3. Biomechanical comparison of Proximal Femoral Nail \& Locking Proximal Anatomic Femoral Plates in Femoral fracture fixation- A study on Synthetic Bones. Indian Journal of Orthopaedics: Vol 49 -issue 3, May - June 2015;247-52.

4. Result of Proximal Femur Nail antirotation for low velocity trochanteric fractures in elderly. Indian Journal of Orthopaedics: Vol 46-issue 5, Sep -Oct 2012;556-61.

5. Calcar Femorale Grafting in the hemiarthroplasty of the hip for unstable intertrochanteric fractures. Indian Journal of Orthopaedics: Vol 49, issue 6, Nov-Dec, 2015;602-10.

6. Comparison of cutout resistance of DCS \& PFN in severe oblique trochanteric fractures. A biomechanical study. Indian Journal of Orthopaedics: Vol 46, issue 3, MayJune 2012;259-65.

7. Campbell's Operative Orthopaedics. 12th edition; Intertrochanteric Fractures 2737-2750.

8. Campbell's Operative Orthopaedics. 13th edition; Intertrochanteric fractures 2829-2840.

9. Rockwood Green. 7th edition; Vol 2, Intertrochanteric Fractures 1597-1637. 